\title{
Pancreatic Neuroendocrine Tumor pT4 TNM Finding v8
}

National Cancer Institute

\section{Source}

National Cancer Institute. Pancreatic Neuroendocrine Tumor pT4 TNM Finding v8. NCI

Thesaurus. Code C135555.

Pancreatic neuroendocrine tumor invading adjacent organs (stomach, spleen, colon, adrenal gland) or the wall of large vessels (celiac axis or the superior mesenteric artery). (from AJCC 8th Ed.) 